\title{
Optimizing the diagnostic capacity for COVID-19 PCR testing for low resource and high demand settings: The development of information-dependent pooling protocol
}

\author{
Damir Vukičević1, Ozren Polašek ${ }^{2}$ \\ ${ }^{1}$ Department of Mathematics, Faculty of \\ Science, University of Split, Split, Croatia \\ ${ }^{2}$ Department of Public Health, University of \\ Split School of Medicine, Split, Croatia
}

\begin{abstract}
Aim To compare different pooling methods in an attempt to improve the COVID-19 PCR diagnostic capacities.

Method We developed a novel information-dependent pooling protocol (indept), based on transmission of less informative sequential pools on to the next pooling cycle to maximize savings. We then compared it to the halving, generalized halving, splitting and hypercube protocols in a simulation study, across variety of scenarios.

Results All five methods yielded various amount of test savings, which mostly depended on the virus prevalence in the population. In situations of low prevalence (up to 5\%), indept had the best performance, requiring on average $20 \%$ of tests needed for singular testing across scenarios that were analyzed. Nevertheless, this comes at the expense of speed, with the worst-case scenario of indept protocol requiring up to twice the time needed to test the same number of samples in comparison to the hypercube protocol. In order to offset this, we developed a faster version of the protocol (indeptSp), which minimizes the number of terminal pools and manages to retain savings compared to other protocols, despite marginally longer processing times.
\end{abstract}

Conclusion The increasing demand for more testing globally can benefit from application of pooling, especially in resource-restrained situations of the low- and middle-income countries or situations of high testing demand. Singular testing in situations of low prevalence should be systematically discouraged.

\section{Correspondence to:}

Ozren Polašek

Department of Public Health

University of Split School of Medicine

Šoltanska 2

21000 Split, Croatia

ozren.polasek@mefst.hr
Pooling is merging of similar biological samples of unknown diagnostic status, in order to optimise the use of laboratory resources. As such, it is of high interest in resource-restraint situations, regardless of the reagents, equipment or time. The emergence of COVID-19 has introduced such a situation globally, with huge increase in the diagnostic demand that requires optimization of laboratory workflow and entire health care [1,2].

The theoretical framework for successful pooling resides in several assumptions, and the principal one is that the pooling will not cause dilution that will devoid the mixture form ability to detect the positive sample from it. Pooling is only meaningful in situations of low prevalence, since an increase in prevalence renders it less effective $[3,4]$. Three main theoretical approaches for pooling exist; halving, generalized halving and 
splitting, which use different scheme to split samples in subsequent pools. In addition, several more approaches were developed to offset specific situations, including a double-averaging model under unknown prevalence [5], double pooling [6], or a multidimensional pooling, which assumes that a sample may enter the testing at multiple stages [7], using multiple combinations [8] and a non-adaptive approaches [9]. Recently, a novel approach was developed, based on the hypercube probing, which was validated and shown substantial savings are feasible, even in low- and middle-income countries [10].

Next important question is the pool size, which was suggested to ideally range between 4 and 10 samples [11-18]. Even larger pools were shown to be effective, with 32 [19-21] or even up to 80 pooled samples that were reported to be effective in laboratory-validated viral diagnostics [22]. Overall, regardless on the size of the pool, previous papers have reported fundamental savings, up to $89 \%$ fewer tests in situations with prevalence under 5\% [23]. When the prevalence increases, pooling may still yield savings over the consecutive testing approach [24]. Finally, field-testing is a critical component of the overall assessment of pooling. Previous studies often did report substantial savings if pooling was applied $[1,10,25]$, but some studies reported lower gains in real situations, compared to theoretical expectations [26,27].

The aim of this study was to compare the most prevalent pooling methods and to optimize the savings by developing a novel, information-dependent protocol.

\section{MATERIAL AND METHODS}

This was a simulation study, based on computer-generated scenarios, with the principal aim of selecting the best pooling protocol available. First, we can define the pool size $(P)$, as the number of initial samples (or swabs) that can be pooled into a single pool. We also define the number of aliquots that can be created from a single biological sample (denoted as $T$ ) and prevalence of virus in the population $(p)$ as the main variables for the study. In addition, a number of initial assumptions must be satisfied (Online Supplementary Document). In addition, three main assumptions were initially put in place: (i) re-testing of the sample testing produces the same result, (ii) if one positive sample in the pool yields the positive result when individually tested, the pool would be positive, and (iii) if all the samples in the pool individually tested give negative results, then the pool would be negative (Online Supplementary Document).

First, we developed a novel information-dependent protocol (indept). This protocol utilizes information from all the tested pools, including negatives and previous pooling cycles, in order to maximize the gains through reduction of the number of test runs. This is done in a multi-dimensional fashion, through transmission of selected, less informative pools on to the next pooling cycle (denoted as $G$ ), where it is possible to optimize the process even further (Figure S4 in the Online Supplementary Document). This protocol can be demonstrated in a simple example, where two samples are pooled and their test result is positive. In the next cycle, we need to test the first sample, which if tested negative, the protocol does not require and more testing, as the second sample is positive. If the first sample is tested positive, then we do not know the status of the second sample. Now, instead of testing the second sample, we relocate it to the next pooling cycle, where we pool it with another similar situation, until we get a pool of negative result. It is imperative to build in the criterion that each biological specimen has a finite number of test runs that can be done from it (number of aliquots), therefore a pooling protocol must not violate this limitation in order to retain diagnostic ability for each biological sample.

We then compared three theoretical protocols, namely halving, generalized halving and splitting with indept (Figures S1-S3 in the Online Supplementary Document). This comparison was performed in the range of scenarios in which the three comparative protocols had the best yields, which was based on the initial pool size of $32(P=32)$. We then compared indept with the recently described hypercube protocol [10]. This comparison favoured the theoretical assumptions for the greatest yields of the hypercube protocol, with the initial pool size of $64(P=64)$. Both groups were compared across a range of prevalence, from 0.1 to $5 \%$, and the aliquot number varying from $T=2$ to $T=6$. The main outcome measure in all instances was the percent of tests that were utilised (PTU), defined as number of tests needed to determine the status of every sample in the pool compared to singular (un-pooled) testing. Notably, savings can be defined as the inverse value of this number.

In addition, we defined the ratio of PTU (rPTU) as the ratio of PTU of indept over the PTU of remaining protocols, for any given prevalence and aliquot size combination. This allowed the direct comparison of savings of two protocols, where the value of 1.0 meant that the two protocols performed similarly, values lower than 1.0 denoted savings while values above 1.0 denoted greater cost of indept compared to the re- 
maining four protocols, in relation to number of tests that were utilized. We also defined the times needed to complete the entire testing cycle. This calculation was based on a simulation of the number of cycles needed to complete the diagnostic process of all samples within the pool (Online Supplementary Document).

All the protocols were developed in C\# and the source code is available upon request. Since no contact was made with any of the patient data or biological samples, no ethical approval was sought. The comparisons of results were made by $t$ test, with significance set at $P<0.05$.

\section{RESULTS}

All of the protocols yielded substantial reduction in the number of tests compared to singular testing. Varying the prevalence from $0 \%-5 \%$ with five possible aliquot numbers suggested the average halving percent of test utilized (PTU) of $0.30 \pm 0.14$ compared to singular sample testing, generalized halving $0.23 \pm 0.09$, splitting $0.22 \pm 0.09$, all for $P=32$ (Table 1). In larger initial pool size, hypercube required $0.24 \pm 0.10$ of singular tests, while indept required only $0.20 \pm 0.10$ tests (Table 1). Notably, the best savings were seen in cases of the lowest prevalence rates; prevalence rise reduced savings across all methods. The savings were lower in cases of lower number of aliquots, with two aliquots requiring $0.35 \pm 0.12$, while six aliquots required $0.19 \pm 0.08$ for $P=32$ (Table 1 ). The full data set is available in the Online Supplementary Document.

Table 1. Percent of test that were saved by pooling (PTS) across protocol used and number of available aliquots

\begin{tabular}{|c|c|c|c|c|c|c|}
\hline \multirow{2}{*}{ Protocol and INITIAL POOL SIZE (P) } & \multicolumn{6}{|c|}{ Number of alouots from a sIngle biological sample (swab) } \\
\hline & $t=2$ & $t=3$ & $t=4$ & $t=5$ & $t=6$ & Average, per protocol \\
\hline \multicolumn{7}{|l|}{$P=32$} \\
\hline Halving & $0.54 \pm 0.02$ & $0.32 \pm 0.04$ & $0.23 \pm 0.06$ & $0.20 \pm 0.09$ & $0.20 \pm 0.09$ & $0.30 \pm 0.14$ \\
\hline Generalized halving & $0.29 \pm 0.10$ & $0.24 \pm 0.09$ & $0.21 \pm 0.08$ & $0.20 \pm 0.08$ & $0.02 \pm 0.09$ & $0.23 \pm 0.09$ \\
\hline Splitting & $0.29 \pm 0.10$ & $0.22 \pm 0.09$ & $0.20 \pm 0.09$ & $0.20 \pm 0.08$ & $0.20 \pm 0.09$ & $0.22 \pm 0.09$ \\
\hline Indept & $0.29 \pm 0.10$ & $0.21 \pm 0.09$ & $0.18 \pm 0.08$ & $0.17 \pm 0.08$ & $0.17 \pm 0.08$ & $0.20 \pm 0.09$ \\
\hline Average, per number of aliquots & $0.35 \pm 0.12$ & $0.25 \pm 0.09$ & $0.21 \pm 0.08$ & $0.19 \pm 0.08$ & $0.19 \pm 0.08$ & - \\
\hline \multicolumn{7}{|l|}{$P=64$} \\
\hline Hypercube & $0.29 \pm 0.10$ & $0.23 \pm 0.10$ & $0.23 \pm 0.10$ & $0.23 \pm 0.10$ & $0.23 \pm 0.10$ & $0.24 \pm 0.10$ \\
\hline Indept & $0.29 \pm 0.10$ & $0.21 \pm 0.09$ & $0.18 \pm 0.08$ & $0.17 \pm 0.08$ & $0.17 \pm 0.08$ & $0.20 \pm 0.10$ \\
\hline Average, per number of aliquots & $0.29 \pm 0.10$ & $0.22 \pm 0.09$ & $0.20 \pm 0.09$ & $0.20 \pm 0.10$ & $0.20 \pm 0.10$ & - \\
\hline
\end{tabular}

The scenario of five aliquots provides the most discriminative power to demonstrate the savings across protocols. The ratio of percentages of tests that were utilized for indept protocol $(r P T U)$ with remaining protocols suggested that indept outperformed all remaining protocols across a variety of scenarios (Figure 1).

We also compared the hypercube and indept in terms of the time needed to analyse all the samples. For this purpose we developed two separate scenarios, one focusing on time needed to detect the negative sam-

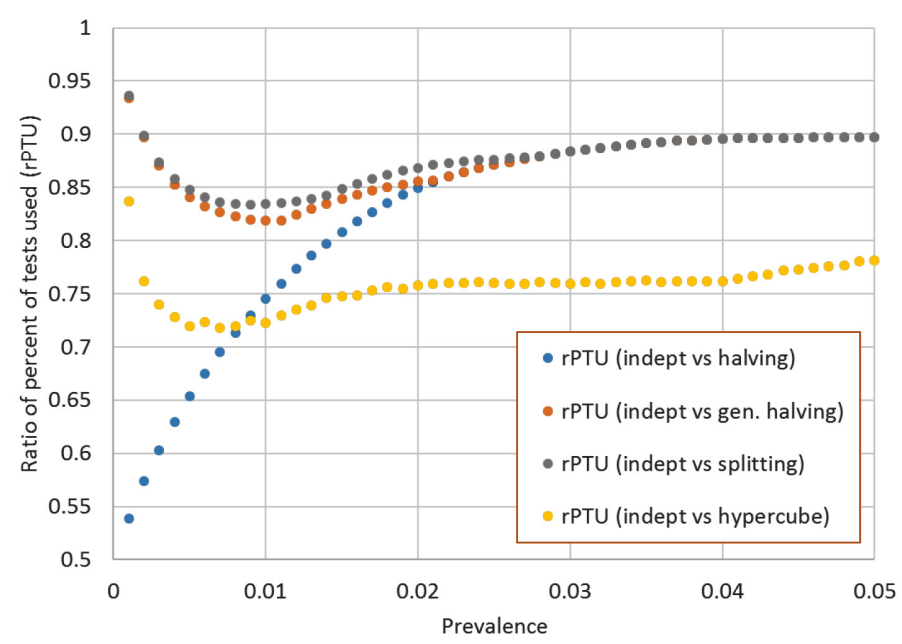

Figure 1. The comparison of ratio of percent of tests used for indept protocol and situation of five aliquots $(T=5)$. ples and the other with time needed to detect positive samples. The initial processing time suggested that indept was requiring nearly twice longer processing times at very low prevalence (0\%-5\%), on average requiring $1.55 \pm 0.18$ more time for detection of positive results, while it required $1.24 \pm 0.14$ more time for detection of negative results (Table S10 in the Online Supplementary Document). In order to offset this difference, we developed indeptSp, a faster version of the protocol, which minimizes the size of terminal pools (Online Supplementary Document). Three thresholds were used, corresponding to an average of $10 \%, 15 \%$ and $20 \%$ longer processing times. This comes at a certain reduction of savings, but manages to retain savings of about $10 \%$, while having 1.08-1.15 times longer detection times for negative cases and 
1.41-1.48 longer times for detection of positive samples, depending on the time extension tolerance (Table S11 in the Online Supplementary Document).

\section{DISCUSSION}

The results of this study demonstrate that indept protocol had the greatest savings among the analysed pooling protocols. The protocol itself is optimized in a way that it does not depend on the assumed regular structure that some other protocols do, therefore enabling the best adaptability and yields. The problem of longer processing times was surpassed by the development of the indeptSp, which minimizes the least informative pools with several samples, which are arbitrarily analysed singularly. This causes certain reduction in total savings, but manages to retain acceptable processing times.

One important issue in comparison of the indept with other protocols was the time needed to detect positive vs negative samples. We believe that assigning negative status should have priority over the positive, since anybody referred to testing must be assumed as positive until proven negative. The development of indeptSp managed to maintain savings, while minimally extending processing times, a strategy that was deemed acceptable [28]. Notably, this may depend on the epidemic spread, since early stages might focus more on detection of positive cases, their quick isolation and contact tracing efforts.

Given the amount of savings demonstrated for each of the protocols, we claim that any population testing in situation of low prevalence (of mainly asymptomatic subjects) should never be tested by singular testing. This is in line with previous studies [10], and it should probably become a norm in the testing laboratories globally, especially if predicted low levels of seropositivity globally are retained [29].

An extension of the idea of pooling could be to establish computer-based algorithms, which will assist the laboratory staff according to the conditions in which the laboratory operates. Based on the data from previous days or weeks, the software could suggest the pooling tool that would be optimal, including savings, pre-processing time and effort, or other metrics that could assist. This might provide the optimisation that would be capable to provide the best achievable savings, which are direly needed in low resource settings [30].

The limitations of this study include the fact that it was a theoretical development without laboratory validation. In addition, this study assumes no substantial errors in the process, and is therefore an optimistic account of the situation, which might prove less efficient in laboratory conditions, where certain level of errors in testing is expected. Nevertheless, this study provides a theoretical benchmark that could be targeted by the future development and subsequently further adjusted to local conditions. The worldwide demand for diagnostic testing is increasing, making any kind of assistance direly needed. This is why we think that any attempt to do this may serve immensely, especially in low- and middle-income countries, where the cost of human labour is lower, but where the access to testing supplies may be lesser. Next step in the protocol development is the field-testing of the idea, aiming to demonstrate the feasibility of this protocol in real-life surroundings.

Funding: OP is supported by the Croatian National Centre of Research Excellence in Personalized Healthcare grant (number KK.01.1.1.01.0010), and the Centre of Competence in Molecular Diagnostics (KK.01.2.2.03.0006).

Authorship contributions: DV and OP are sole authors.

Competing interests: OP is scientific advisor to the Government of the Republic of Croatia for COVID-19 response, which had no influence in the decision to prepare the manuscript, its preparation or the selection of the journal where the manuscript would be submitted. The authors completed the ICMJE Unified Competing Interest form at (available upon request from the corresponding author), and declare no further conflicts of interest.

\section{Additional material}

Online Supplementary Document

1 Garg J, Singh V, Pandey P, Verma A, Sen M, Das A, et al. Evaluation of sample pooling for diagnosis of COVID-19 by real timePCR: A resource-saving combat strategy. J Med Virol. 2020. Online ahead of print. Medline:32869865 doi:10.1002/jmv.26475

2 Sekulić A, Likić R, Matas M. How to allocate intensive care resources during the COVID-19 pandemic: medical triage or a priori selection? Croat Med J. 2020;61:276-8. Medline:32643345 doi:10.3325/cmj.2020.61.276

3 Nalbantoglu OU. Group testing performance evaluation for SARS-CoV-2 massive scale screening and testing. BMC Med Res Methodol. 2020;20:176. Medline:32615934 doi:10.1186/s12874-020-01048-1 
4 Regen F, Eren N, Heuser I, Hellmann-Regen J. A simple approach to optimum pool size for pooled SARS-CoV-2 testing. Int J Infect Dis. 2020;100:324-6. Medline:32866638 doi:10.1016/j.ijid.2020.08.063

5 Pikovski A, Bentele K. Pooling of coronavirus tests under unknown prevalence. Epidemiol Infect. 2020;148:e183. Medline:32758313 doi:10.1017/S0950268820001752

6 Broder AZ, Kumar R. A note on double pooling tests (preliminary version). arXiv. 2020:2004.01684v1. Available: https://arxiv. org/pdf/2004.01684.pdf. Accessed: 28 December 2020.

7 Pilcher CD, Westreich D, Hudgens MG. Group Testing for Severe Acute Respiratory Syndrome- Coronavirus 2 to Enable Rapid Scale-up of Testing and Real-Time Surveillance of Incidence. J Infect Dis. 2020;222:903-9. Medline:32592581 doi:10.1093/ infdis/jiaa378

8 Shental N, Levy S, Wuvshet V, Skorniakov S, Shalem B, Ottolenghi A, et al. Efficient high-throughput SARS-CoV-2 testing to detect asymptomatic carriers. Sci Adv. 2020;6:eabc5961. Medline:32917716 doi:10.1126/sciadv.abc5961

9 Täufer M. Rapid, large-scale, and effective detection of COVID-19 via non-adaptive testing. J Theor Biol. 2020;506:110450. Medline:32814072 doi:10.1016/j.jtbi.2020.110450

10 Mutesa L, Ndishimye P, Butera Y, Souopgui J, Uwineza A, Rutayisire R, et al. A pooled testing strategy for identifying SARSCoV-2 at low prevalence. Nature. 2020. Online ahead of print. Medline:33086375 doi:10.1038/s41586-020-2885-5

11 Khodare A, Padhi A, Gupta E, Agarwal R, Dubey S, Sarin SK. Optimal size of sample pooling for RNA pool testing: An avant-garde for scaling up severe acute respiratory syndrome coronavirus-2 testing. Indian J Med Microbiol. 2020;38:18-23. Medline:32719204 doi:10.4103/ijmm.IJMM_20_260

12 Kim SY, Lee J, Sung H, Lee H, Han MG, Yoo CK, et al. Pooling Upper Respiratory Specimens for Rapid Mass Screening of COVID-19 by Real-Time RT-PCR. Emerg Infect Dis. 2020;26:2469-72. Medline:32844739 doi:10.3201/eid2610.201955

13 Perchetti GA, Sullivan KW, Pepper G, Huang ML, Breit N, Mathias P, et al. Pooling of SARS-CoV-2 samples to increase molecular testing throughput. J Clin Virol. 2020;131:104570. Medline:32805524 doi:10.1016/j.jcv.2020.104570

14 Lim KL, Johari NA, Wong ST, Khaw LT, Tan BK, Chan KK, et al. A novel strategy for community screening of SARS-CoV-2 (COVID-19): Sample pooling method. PLoS One. 2020;15:e0238417. Medline:32857823 doi:10.1371/journal.pone.0238417

15 Praharaj I, Jain A, Singh M, Balakrishnan A, Dhodapkar R, Borkakoty B, et al. Pooled testing for COVID-19 diagnosis by real-time RT-PCR: A multi-site comparative evaluation of 5- \& 10-sample pooling. Indian J Med Res. 2020;152:88-94. Medline:32893844 doi:10.4103/ijmr.IJMR_2304_20

16 Abdalhamid B, Bilder CR, McCutchen EL, Hinrichs SH, Koepsell SA, Iwen PC. Assessment of Specimen Pooling to Conserve SARS CoV-2 Testing Resources. Am J Clin Pathol. 2020;153:715-8. Medline:32304208 doi:10.1093/ajcp/aqaa064

17 Alcoba-Florez J, Gil-Campesino H, García-Martínez de Artola D, Díez-Gil O, Valenzuela-Fernández A, González-Montelongo R, et al. Increasing SARS-CoV-2 RT-qPCR testing capacity by sample pooling. Int J Infect Dis. 2020;103:19-22. Medline:33220439 doi:10.1016/j.ijid.2020.11.155

18 Smalley DL, Cisarik PM, Grantham J, Cloud W, Neil RB, DePriest P. Impact of Pool Testing in Detection of Asymptomatic Patients with COVID-19. Lab Med. 2020. Online ahead of print. Medline:33206195 doi:10.1093/labmed/lmaa094

19 Yelin I, Aharony N, Shaer Tamar E, Argoetti A, Messer E, Berenbaum D, et al. Evaluation of COVID-19 RT-qPCR test in multi-sample pools. Clin Infect Dis. 2020;71:2073-8. Medline:32358960 doi:10.1093/cid/ciaa531

20 Sunjaya AF, Sunjaya AP. Pooled Testing for Expanding COVID-19 Mass Surveillance. Disaster Med Public Health Prep. 2020;14:e42-e43. Medline:32660684 doi:10.1017/dmp.2020.246

21 Rai B, Shukla A, Choudhary G, Singh A. Pool Testing for COVID-19: Suitable Splitting Procedure and Pool Size for India. Disaster Med Public Health Prep. 2020;1-17. Medline:32907662 doi:10.1017/dmp.2020.335

22 Petrovan V, Vrajmasu V, Bucur AC, Soare DS, Radu E, Dimon P, et al. Evaluation of Commercial qPCR Kits for Detection of SARS-CoV-2 in Pooled Samples. Diagnostics (Basel). 2020;10:472. Medline:32664511 doi:10.3390/diagnostics10070472

23 Deckert A, Bärnighausen T, Kyei NN. Simulation of pooled-sample analysis strategies for COVID-19 mass testing. Bull World Health Organ. 2020;98:590-8. Medline:33012859 doi:10.2471/BLT.20.257188

24 Brynildsrud O. COVID-19 prevalence estimation by random sampling in population - optimal sample pooling under varying assumptions about true prevalence. BMC Med Res Methodol. 2020;20:196. Medline:32703158 doi:10.1186/s12874-02001081-0

25 Gupta E, Padhi A, Khodare A, Agarwal R, Ramachandran K, Mehta V, et al. Pooled RNA sample reverse transcriptase real time PCR assay for SARS CoV-2 infection: A reliable, faster and economical method. PLoS One. 2020;15:e0236859. Medline:32730368 doi:10.1371/journal.pone.0236859

26 Singh AK, Nema RK, Joshi A, Shankar P, Nema S, Raghuwanshi A, et al. Evaluation of pooled sample analysis strategy in expediting case detection in areas with emerging outbreaks of COVID-19: A pilot study. PLoS One. 2020;15:e0239492. Medline:32960929 doi:10.1371/journal.pone.0239492

27 Lüsebrink J, Schildgen V, Schildgen O. Pooling is an insufficient strategy to avoid health care staff to patient transmission of SARS-CoV-2. Infect Control Hosp Epidemiol. 2020;1-3. Medline:33213555 doi:10.1017/ice.2020.1340

28 Denny TN, Andrews L, Bonsignori M, Cavanaugh K, Datto MB, Deckard A, et al. Implementation of a Pooled Surveillance Testing Program for Asymptomatic SARS-CoV-2 Infections on a College Campus - Duke University, Durham, North Carolina, August 2-October 11, 2020. MMWR Morb Mortal Wkly Rep. 2020;69:1743-7. Medline:33211678 doi:10.15585/mmwr.mm6946e1

29 Rostami A, Sepidarkish M, Leeflang MMG, Riahi SM, Nourollahpour Shiadeh M, Esfandyari S, et al. SARS-CoV-2 seroprevalence worldwide: a systematic review and meta-analysis. Clin Microbiol Infect. 2020. Online ahead of print. Medline:33228974 doi:10.1016/j.cmi.2020.10.020

30 Deka S, Kalita D. Effectiveness of Sample Pooling Strategies for SARS-CoV-2 Mass Screening by RT-PCR: A Scoping Review. J Lab Physicians. 2020;12:212-8. Medline:33268939 doi:10.1055/s-0040-1721159 